\title{
Measurement of bistability in a multidimensional parameter space
}

\author{
Vincent Jaquet $^{\mathrm{a}}$, Chieh Hsu ${ }^{\mathrm{a}, \mathrm{b}}$ \& Attila Becskei ${ }^{\mathrm{a}}$ \\ ${ }^{a}$ Biozentrum, University of Basel, Klingelbergstrasse 50/70, 4056 Basel, Switzerland \\ ${ }^{\mathrm{b}}$ School of Biosciences, University of Kent, Canterbury, Kent CT2 7NJ, UK
}

To whom correspondence should be addressed. E-mail: attila.becskei@unibas.ch

Transition rate / bistability / positive feedback loop / synthetic gene network

\begin{abstract}
Bistability plays an important role to generate two stable states for alternative cell fates, or to promote cellular diversity and cell cycle oscillations. Positive feedback loops are necessary for existence of bistability and ultrasensitive reactions in the loops broaden the parameter range of bistability. The broader parameter range a system's bistability covers, the more robust the two states are. It is challenging to determine the bistable range of a parameter because noise and transient processes induce transitions between the two states. We found that a threshold of transition rates coincides with the bistability boundaries determined by the open-loop approach. With this threshold, we estimated the boundaries for various synthetic single-gene positive feedback loops in yeast in a two dimensional parameter space: the inducer concentration and promoter dynamic range. While the bistable range of inducer concentration was influenced by many factors, the promoter dynamic range was more informative. The narrowest promoter dynamic range at which bistability can emerge revealed whether the full potential of an ultrasensitive reaction, such as dimerization, is exploited in the feedback loop. The convenient control of basal expression to adjust the promoter dynamic range permits a practical and reliable comparison of robustness of related positive feedback loops.
\end{abstract}

\section{Introduction}

Positive feedback loops are recurring patterns in genetic regulatory network as their ability to generate bistability - the maintenance of two stable expression states under identical conditions is broadly found in many biological processes like cell-fate determination, spatial organization of cellular components during cell polarization, cell division or cancer onset ${ }^{1-8}$. For a single feedback loop to be bistable, its reactions must generate a non-linear switch-like sigmoidal response, termed ultrasensitive response even in the absence of feedback regulation ${ }^{9-11}$. In the absence of ultrasensitive reactions, the feedback loop is strictly monostable, and the expression converges to a single steady-state level. Reactions like sequestration by inhibitor molecules, cooperative binding and dimerization can generate an ultrasensitive response ${ }^{12}$.

In order to function robustly, bistability has to be maintained when parameter values change. Usually, the robustness of bistability is examined in a single dimension of the parameter space, 
exemplified by the variation of the concentration of an inducer molecule to adjust the binding affinity of the transcription factor (TF) to the DNA. Another, more rarely studied parameter is the dynamic range of the promoter, which is the ratio of the maximal to basal expression of a gene driven by the promoter. The promoter dynamic range can be conveniently controlled experimentally by tuning the basal expression, also known as leakiness ${ }^{13}$.

The combined analysis of the bistable domain of these two parameters can be very informative to characterize the robustness of positive feedback loops. Bistability can emerge when the promoter dynamic range increases beyond a critical (extremal) value. Each prototypical feedback loop has a specific extremal value. For example, the promoter dynamic range has to be eight or larger when dimerization is the only ultrasensitive reaction in the feedback loop ${ }^{12}$. On the other hand, bistability can emerge even when the promoter dynamic range is less than 8 when ultrasensitivity is due to sequestration of the activator in the feedback loop.

It is not trivial to determine the bistability range of a parameter experimentally. Bistability is a deterministic and equilibrium concept while gene expression is noisy and is described stochastically. Furthermore, processes with long transient kinetics, such as the slow accumulation of the inducer in the cell. Noise and slow transient processes induce transitions between two expression states. Such transitions occur both inside and outside of the bistable range of a parameter, at varying rates. Thus, the steady-state bistability cannot be determined unequivocally.

In our previous study, we showed that the open-loop approach obviates this problem because the open-loop is much less sensitive to noise and transients and therefore, it can precisely delimit the bistable range of a parameter ${ }^{14}$. Yet, it is not trivial to construct the corresponding genetic components, which have to fulfill the criteria required for the open-loop approach, and multiple series of mRNA measurements have to be performed.

Here we present an approach that originates in the observation that the transition rates were relatively constant at the bifurcation points in related feedback loops. This constancy is in part due to the fact that transient kinetics affects the transitions mostly outside of the bistable range while noise inside the bistable range, leaving the bistability boundary relatively unaffected ${ }^{14}$. We measured the transition rates between the two states and defined a threshold value to approximate the bistable range that was validated with the open-loop predictions. Thus, the transition-rate based approach allowed the comparison of related feedback loops, without the need to create additional open-loop constructs.

\section{Results}

\section{The transition rate at the bistability boundaries in model feedback loops}

We aimed to assess how the measurement of the transition rate can be used to map the bistability boundaries in a multidimensional parameter space. For this purpose, we explored a model of a simple feedback loop, in which a TF binds cooperatively to the promoter of its own gene (Fig. 1B). First, we plotted the steady-state expression levels of a deterministically defined system. To 
define the range of bistability in the deterministic system, the TF-DNA affinity was varied. The bistable range of this parameter is flanked by monostable ranges, in which either only the lower or the higher state exists (Fig. 1A). The basal expression reduces the bistable range of the TFDNA affinities. When it passes a critical value, bistability is eliminated altogether (Fig. 1A). This critical value is characteristic for different systems and can be expressed by dimensionless numbers when the basal expression is normalized by the maximal expression of the promoter, which yields the relative basal expression. The relative basal expression is the reciprocal of the promoter dynamic range. The critical value of the relative basal expression is $1 / 8$ in the above system, with a Hill-coefficient $=2$.

Since gene expression is noisy, noise induces transitions between the two states ${ }^{14-17}$. The speed of the transitions is quantified by the transition rate, which is the inverse of the time required to escape a state, i.e. the mean first-passage time (MFPT). To see how the transition rates relate to the bistability boundaries and how this is influenced by the basal expression, we performed a stochastic simulation of the feedback loop (Fig. 1C), using the Gillespie stochastic simulation algorithm. The feedback loop was parametrized with specific, realistic, mRNA and protein degradation rates. The rate of the transitions from the high and low expression states (ON-toOFF transitions) declined precipitously in the vicinity of the bistable boundaries. For the OFF-toON transitions, the rates also decreased toward the interior of the bistable range although less abruptly. This more gradual decline is due to the larger noise in the OFF state, which arises due to the small number of molecules ${ }^{14,18}$.

Interestingly, both the OFF-to-ON and the ON-to-OFF transition rates intersected the bistable boundaries at a value of around $0.02 \mathrm{~h}^{-1}$, which corresponds to a mean passage time of two days. This value is relatively constant even when the basal expression was varied to modulate the promoter dynamic range. Thus, single threshold value can be used in good approximation to delimit the bistable range when basal expression is low or high (Fig. 1C). The same transition rate threshold can also be used to estimate the bistability range with a small error when the cooperativity of the TF binding was varied within realistic range (Hill-coefficient between 1.5 and 3, Fig. S1).

When the basal expression is increased, the two bistable boundaries approach each other and coalesce at an extremal point, the cusp point, which marks the extreme point of bistability (Fig. 1D). To determine the location of the cusp point, we plotted the estimated bistability boundaries in TF-DNA affinity dimension for each measured basal expression in a log-log plot. When these points are connected and extrapolated by linear regression, the two lines delimited an area that is very similar to the bistable domain obtained by the bifurcation analysis (Fig. 1D, dashed and full lines). Thus, measuring the transition rates and applying a threshold on them is expected to approximate the bistable domain and its extremal point, the cusp point.

Estimation of the bistable domain in a two-dimensional parameter space with a threshold value of the transition rate 
To test the above approach, we constructed yeast cells that contain synthetic feedback circuits. Synthetic circuits have played a main role in understanding the principles of cellular dynamical behavior $^{19-26}$. In the synthetic positive feedback loops, the TF binds to the promoter of its own gene (Fig. 2A). We inserted RNA stem-loop sequences upstream of the start codon of the TF to reduce the translation efficiency and the cellular concentration of the transcriptional activator (Table S1). This is important to prevent cellular growth defects due to the overexpressed transcriptional activator. The employed stem loops reduce translation considerably, up to around 100 times $^{27}$. At first, we used the tet-transcriptional activator (tTA) as a TF, also known as the tet-OFF system ${ }^{28}$. It binds to the tet operator as a dimer. When doxycycline is added it dissociates from the tet operator. In this way, doxycycline can be used to adjust the apparent affinity of the tTA to the tet operator (TF-DNA affinity).

We extended the feedback circuits with an additional copy of the tTA gene under the control of the $G A L$ promoter to adjust the basal expression and to set the initial conditions. We combined these two aims by controlling the GAL promoter with two related transcriptional activators, Gal4p and GEV (see Methods). The endogenous Gal4p was used to set the initial condition. If the cells are transiently exposed to galactose, the initial condition corresponds to the high expression state. Consequently, the ON-to-OFF transitions can be measured. Without pre-exposing the cells to galactose, the OFF-to-ON transitions can be measured. GEV is a synthetic transcriptional activator consisting of the Gal4 DNA binding domain, and estradiol receptor and the VP16 activation domain. The GEV was employed to adjust the basal expression because it modulates gene expression in a graded way in response to estradiol ${ }^{14}$. The basal expression was quantified by measuring the mRNA encoding the TF (see Methods).

As mentioned in the introduction, positive feedback loop displays bistability, only if it incorporates ultrasensitive reactions. The homodimerization of tTA may in principle be sufficient to generate bistability. However, combining cooperative binding with dimerization can expand the bistable range. Cooperative binding can occur when there are multiple binding sites in the promoter. Therefore, we inserted promoters with a single or multiple binding sites in the gene circuit.

Upon the initial characterization of the circuits (Fig. S2), we measured both the OFF-to-ON and ON-to-OFF transition rates as the doxycycline concentration was varied (Fig. 2B). We interpolated the measured OFF-to-ON and the ON-to-OFF transition rates with linear and power regression, respectively (see Methods). Next, we applied a threshold at $0.02 \mathrm{~h}^{-1}$. The intersection of the threshold with the interpolation curves defines the doxycycline concentrations that mark the boundaries of the presumptive bistable range of the inducer concentration.

Next, we investigated how the promoter dynamic range affects the bistable range of the inducer concentration. The OFF-to-ON transitions became faster as the basal expression was increased. On the other hand, the ON-to-OFF transitions remained largely unaffected by variations in basal expression, as expected from the simulations (Fig. 1C, D). The estimated bistability boundaries were plotted as a function of basal expression and these points were extrapolated by linear 
regression in the logarithmic plots to delimit the presumptive bistable domain in the twodimensional parameter space (relative basal expression and apparent TF-DNA affinity) (Fig. 3A). Increasing basal expression narrowed the bistable range of doxycycline concentrations. The presumptive bistable domain was larger for the circuit with multiple tet operators than with a single operator, in accordance with the expectations.

\section{Validation of the presumptive bistable domains with the open-loop approach}

Next, we examined feedback loops in which tTA is replaced by rtTA. rtTA is a mutant form of tTA and displays a doxycycline-dependent behavior opposite to rtTA: it binds to the tet operators in the presence of doxycycline (also termed the tet-ON system). For these feedback circuits, published open-loop data are available, which can be used to identify bistable boundaries directly $^{14}$. In brief, a component in the feedback loop is broken into an input and output using the conditions specified in the experimental open-loop approach. This creates a reaction chain starting by the input passing through all the components of the broken loop and ending at the output (Fig. 4A). The open-loop function is fitted to data pairs consisting of the input and output expression levels, at different doxycycline concentrations. Here we calculated the bistability boundaries for two circuits, both of them contain seven operators in the promoter. One of the circuits contains the naturally dimeric rtTA. The other circuit contains a monomeric form of rtTA, sc-rtTA. Since the dimeric circuit incorporates two ultrasensitive reactions, cooperative binding and dimerization, it has a broader bistability range in the TF-DNA affinity dimension.

When we compared the calculated bistability boundaries based on the open-loop approach (dashed curves in Fig. 3B) with the presumptive bistability boundaries based on the transition rate threshold (full curves in Fig. 3B, Fig. S3), a good agreement was observed for both the monomeric and dimeric circuits. The relative basal expression at the cusp point, where the two bistable boundaries intersect, was around 0.1 for sc-rTA using both methods. This value was three times higher, around 0.3 , for rtTA.

\section{Comparison of measured and predicted transition rates}

For the rtTA circuits, parameters and noise values are known ${ }^{14}$, and the transition rates can be predicted by a stochastic simulation and compared to experimental data. The predictions are in good agreement with the experiments, particularly for the sc-rtTA circuit (see Methods for explanation). The transition rates become faster as the basal expression is increased. The bands representing the rates of equal values run approximately parallel to the bistability boundaries (Fig. 3C,D).

\section{Differential robustness of bistability in the two dimensions of the parameter space}

We compared the bistable boundaries of the dimeric rtTA and monomeric sc-rtTA circuits in the two parameter dimensions. At the endogenous basal expression of the feedback circuits (leftmost points in Fig. 3B), the bistable range of the doxycycline concentration is larger than hundredfold for rtTA and it is merely twofold for sc-rtTA (Fig. 5C). However, the endogenous basal 
expression is different for the two circuits and with increasing basal expression the bistable inducer range narrows down. Thus, a faithful comparison can be made at equal basal expression. Therefore, we calculated the interpolated bistable range at the same basal expression (Fig. 5C). The difference between the two circuits became smaller but was still very large. On the other hand, the relative basal expression at the cusp point differs only by a factor of 4.5 between the two circuits.

How is it possible that for the sc-rtTA, the bistability is much more robust in the relative basal expression dimension than in the doxycycline/TF-DNA affinity dimension?

To understand this peculiar behavior, it is important to distinguish two entities: system variables and system parameters. The TF concentration is a system variable since it is the TF that activates itself auto-catalytically. The doxycycline modifies to what extent the TF activates its own expression and thus can be viewed as a signal that determines a system parameter. The response of an open system is typically measured in response to the signal. However, the signal (in this case the doxycycline) captures only one of the two ultrasensitive reactions in the $\mathrm{P}_{[\text {tetO]7 }}$-rtTA feedback loop. Since doxycycline influences only the binding of the TF to the promoter but not dimerization, the output in response to doxycycline reports only a single ultrasensitive reaction, the cooperative binding. Only a fully opened feedback loop with an input / output pair captures both ultrasensitive reactions (Fig. 4A).

In the case for the $\mathrm{P}_{[\mathrm{tetO}] 7}$-sc-rtTA, only cooperative binding constitutes an ultrasensitive reaction. Thus, the open-loop output has very similar responses with respect to the input and the doxycycline and therefore their fitted Hill-coefficients for these two functions are nearly equal (1.94 and 1.90) ${ }^{14}$ (Fig. 4B,C). On the other hand, the two fitted Hill-coefficients are quite different for rtTA, i.e. 2.89 for the apparent Hill coefficient with respect to the input variable and 1.32 for the response to the signal (dox) (Fig. 4B,C). It is also interesting that the binding of scrtTA to the seven tet operators is, for unknown reasons, more cooperative than that of rtTA (1.94 versus 1.46).

Interestingly, the Hill coefficients of the two functions have different impact on the bistable domain. The Hill-coefficient of the open-loop function with respect to the input expands the bistable domain in both dimensions: the TF-DNA affinity and the relative basal expression (Fig. 4D). On the other hand, the Hill-coefficient of the response to the dox signal does not affect the value of the relative basal expression at the cusp point but it decreases the bistability range with respect to the TF-DNA affinity (Fig. 4E).

Thus, based on these two factors it is possible to explain why the bistable range with respect TFDNA affinity is much larger for rtTA than for sc-rtTA. The large Hill-coefficient of the open-

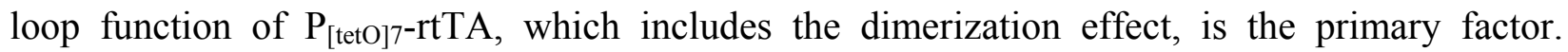
Secondarily, the Hill-coefficient with respect to the signaling molecule is lower for rtTA than for sc-rtTA which further expands the bistable range in the TF-DNA affinity dimension for rtTA. 
This counterintuitive relation has also an impact on the transition rates, as well. The rates decline less rapidly in the $\mathrm{P}_{[\text {tetO }] 7}-\mathrm{rtTA}$ circuit than in the $\mathrm{P}_{[\text {tetO }] 7}-$ sc-rtTA circuit as the TF-DNA affinity is tuned to the interior of the bistable domain (see the narrower bands in Fig. $3 \mathrm{C}$ in comparison to Fig. 3D, below the upper bistable boundaries). This is interesting because the open-loop function is steeper for $\mathrm{P}_{[\operatorname{tetO}] 7}-\mathrm{rtTA}$ than for $\mathrm{P}_{[\text {tetO]7 }}-$ sc-rtTA.

\section{The critical promoter dynamic range required for the emergence of bistability}

Unlike the bistable range of the inducer concentrations, the cusp point was not sensitive to the difference of the response of the output to the input and the signal. To see how general this behavior is, we derived a general formula based on the maximum sensitivity of the open-loop function of a circuit in which a monomeric TF binds to the promoter of its own gene cooperatively. Interestingly, the critical point of the relative basal expression $\left(b / V_{\max }\right)$ was independent of the TF-DNA affinity $\left(K_{\mathrm{d}}\right)$; this critical value depends only on the Hill coefficient with respect to the input, which describes the degree of cooperative binding. When $n>1$, then the binding is cooperative (equation 3).

Plotting this relation reveals that it increases sharply when the Hill-coefficient increases from one and levels off at larger values of $n$. When the TF also acts negatively on the promoter, so that a double positive - negative feedback arises, a similar relation is obtained but the critical relative basal expression has lower values (Fig. 5A,B, Methods).

In more complex feedback loops, the location of cusp (critical) point depends on multiple parameters $^{12}$. In our circuits, the dimerization of the TF has to be taken into account. The weaker the $\mathrm{TF}$ dimerizes, the stronger the ultrasensitivity appears. More precisely, if the protein concentration of the TF is below the dimerization dissociation constant the maximal potential of dimerization to generate bistability is equal to the TF that binds cooperatively with a Hill coefficient of two ${ }^{12}$.

We plotted the $b / V_{\max }$ values at the extrapolated cusp point along this function for the four circuits measured so far. Furthermore, we extrapolated cusp points for $\mathrm{P}_{[\text {tetO]1-rtTA feedback loop }}$ (Fig. 5A). It becomes evident that there are two clusters of points (Fig. 5B). For the two circuits

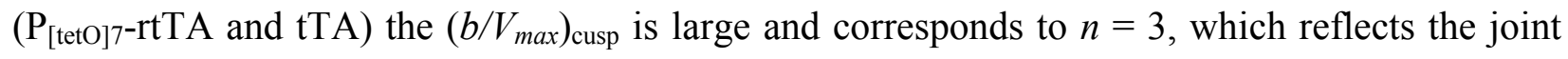
effect of dimerization and cooperative binding. For the other cluster, $\left(b / V_{\max }\right)_{\text {cusp }}$ is less, around 0.1 and the bistability is supported by a single ultrasensitive reaction: cooperative binding or dimerization. The minimal promoter dynamic range to generate bistability by a maximally ultrasensitive dimerization reaction is 8 . Thus, the promoter dynamic ranges of around 10 at the

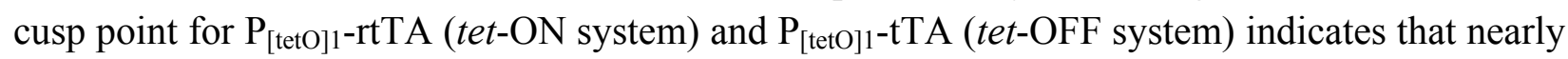
the full potential of dimerization is exploited to generate bistability. For tTA (tet-OFF system), we confirmed that the open-loop response generates a Hill-coefficient 2 (Fig. S4), in agreement with the location of the cusp point. 
Thus, the promoter dynamic range at the extremal point of the bistable domain provides a simple, easily comparable measure of the robustness of bistability.

\section{Discussion}

In this work, we presented an approach to map bistability in a two-dimensional parameter space by setting a threshold in the transitions rates. This value was obtained by simulating the transition rates for a simple feedback loops at the bifurcation boundaries. This rate was relatively constant when the basal expression and cooperativity of binding was varied. Applying this threshold to the variants of the feedback loop, we obtained their presumptive bistability boundaries. This was confirmed with the open-loop approach, which can delimit bistable range directly.

Our transition-rate based approach represents an intermediate between hysteresis experiments and loop opening. Hysteresis experiments and its quantitative versions can map bistable ranges without the need to devise new constructs and to know any of the system parameter, provided some conditions are met ${ }^{14,29,30}$. When transient kinetics and noise is prominent, as in our synthetic circuits, the hysteresis profiles cannot delimit the bistability boundaries unequivocally, In this case, the open-loop approach can be used, which requires new genetic constructs and mRNA measurements ${ }^{14}$.

Often the bistability of multiple feedback loops is compared, which can be versions of synthetic loops or mutant forms of endogenous feedback loops. If one of the feedback loops is characterized by the open-loop approach, it can be used to validate a threshold in the transition rates suitable to define the bistable range in related feedback loops. To explore how this threshold can be used when the promoter dynamic range and the cooperativity (i.e. the degree of ultrasensitivity) is varied, we simulated analogous feedback loops with known mRNA and protein degradation rates. In this way, it is possible to compare the bistability domains of related feedback loops, without the need to construct and measure additional open-loop constructs.

We mapped bistability in a two-dimensional parameter space, which permits a more succinct characterization and faithful comparison of robustness of bistability in related feedback loops. Typically, the bistable range of the inducer concentration is measured in hysteresis experiments, which has several caveats when used to compare different feedback loops. In principle, the bistable range of an inducer can be compared at identical promoter dynamic range since usually the bistable range is increasing with increasing promoter dynamic range. Contrary to the expectations, the promoter dynamic range differs among feedback loops, often due to the variations in the basal expression. For example, changing the number of TF binding sites to modulate cooperative binding can cause inadvertent alterations in the basal expression, which is often difficult to predict ${ }^{31}$. We have evidenced similar alterations in the circuits we explored in this study (compare leftmost points, which represent the basal expression inherent to the feedback circuits, in Fig. 3A,B and 5A,C). When we interpolated the bistability boundaries, we were able to compare bistability ranges at equal relative basal expression. The difference in the bistable 
ranges of the TF-DNA affinity between the dimeric and monomeric circuits became less prominent in this way.

We also found that the cusp (critical) point characterizes robustness more succinctly and faithfully because the promoter dynamic range is directly related to the systems variable in a genetic feedback and it is not distorted by the differential open-loop response to the inducer signal and system variable. Noticeably, two distinct groups of cusp points were found for the loops in this study (Fig. 5B, C) according to the number (one or two) of ultrasensitive reactions in the loop. The feedback loops with a single ultrasensitive reaction (either cooperativity or dimerization) generated bistability only if the promoter dynamic range is larger than 10 . When the two reactions are combined bistability is possible even when the promoter dynamic range is reduced to around 3. While most transcription factors are dimers, this alone does not guarantee ultrasensitivity. The ultrasensitivity increases when the concentration of the total protein concentration is sufficiently low and the maximal ultrasensitivity is attained when the relevant range of protein concentration is below the dimerization dissociation constant. At this case bistability can emerge when the promoter dynamic range is at least 8 . The experimentally observed range was 10; this indicates that nearly the full potential of dimerization is exploited to promote bistability (Fig. 3A,B and 5B).

Thus our approach, which identifies the critical point of bistability using the threshold values of transition rates, is expected to be applicable for various feedback loops to reliably characterize their robustness of bistability, permitting the practical and faithful comparison of different circuits. 


\section{Methods \\ Design of yeast strains}

The feedback circuits were inserted into the chromosome of S. cerevisiae W303 (Table S1). Each strain contains three additional gene constructs: a fluorescent reporter $\left(\mathrm{P}_{[\text {tetO]2-yEGFP), a }}\right.$ construct expressing constitutively GEV transcriptional activator and the $\mathrm{P}_{\mathrm{GAL}}$-rtTA/sc-rtTA/tTA tunable basal expression construct ${ }^{27}$. The expression of the florescent reporter was measured by flow cytometer. The strains for the open-loop construct (Fig. S3) were built as previously described $^{14}$. The number of the AT pairs in the 5 or 6 bp long stem-loop (SL) is indicted in the subscript (Table S1).

\section{Measurement of promoter dynamic range}

The relative basal expression is the ratio of the basal expression to the maximally induced expression in the feedback strain. The expression levels were quantified by qPCR upon RNA isolation as previously described ${ }^{27}$. The cells were grown for $24 \mathrm{~h}$ after setting the appropriate initial condition at 30C in synthetic media containing the indicated concentration of estradiol. For the tet-ON systems (sc-rtTA, rtTA), the basal expression was measured after growing cells in the absence of doxycycline. For the maximally induced expression, cells were grown in media with $19.5 \mu \mathrm{M}$ doxycycline. For the tet-OFF system (tTA feedback loops), $0.2 \mu \mathrm{M}$ doxycycline was used for the basal expression and $0 \mu \mathrm{M}$ for the maximally induced expression. The maximally

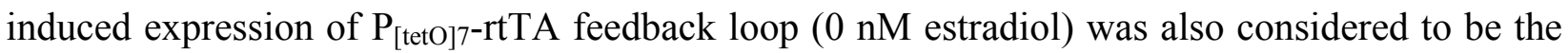
maximal expression for the dual positive-negative feedback loop.

\section{Determination of presumptive bistable boundaries by setting a threshold on the measured}

\section{transition rates}

To assess the transition rates, the proportion of the OFF and ON cells was measured at 7.5, 24, 48 and $72 \mathrm{~h}$ (OFF-to-ON transitions) or 24, 48 and $72 \mathrm{~h}$ (ON-to-OFF transitions) after the initial condition was set. To adjust the initial conditions with the high expression state for tTA, the cells were induced with $0.03 \%$ galactose overnight. For the low expression state the cells were grown overnight in the presence of $0.2 \mu \mathrm{M}$ doxycycline to prevent the binding of tTA to the tet operators. Cells from these cultures were then inoculated into media containing the indicated concentration of doxycycline and estradiol. Prior to the inoculation, cells were washed only for the cultures that were measured after $7.5 \mathrm{~h}$.

The analogous conditions for rtTA were described earlier ${ }^{14}$. The transition rates were fitted by nonlinear regression from the above time series.

To approximate the dependence of the transition rate on the doxycycline concentration, linear regression with inverse-square $\left(\mathrm{Y}^{-2}\right)$ weighting and power regression without weighting was used for the OFF-to-ON and ON-to-OFF transition rates, respectively. For the tTA feedback loops, transition rates measured at a doxycycline concentration below $0.0028 \mu \mathrm{M}$ were not considered for the regression as their values were similar to the ones in absence of doxycycline. 
When the transition rates dropped abruptly as the doxycycline was varied, the rate for only one doxycycline concentration could be obtained in the realistic, measurable, range of ON-to-OFF transition rates. In such cases, the power of the approximating function was set to be equal to the value obtained for $\mathrm{P}_{[\mathrm{tetO}] 7}-\mathrm{rtTA}$ at $0 \mathrm{nM}$.

Subsequently, we applied a threshold at $0.02 \mathrm{~h}^{-1}$ to these approximating functions to obtain the doxycycline concentration for the presumptive bistability boundary. For each boundary value, we measured the relative basal expression. The Log10 transform of these data pairs were used for linear regression to interpolate the boundaries of the bistable domain (see Fig. 3A,B and 5A).

\section{Stochastic simulations of transition rates}

In order to obtain a realistic value for the transition rate at the bistable boundary, we performed a stochastic simulation of simple positive feedback loop mediated by a monomeric TF (Fig. 1 and Fig. S1).

$$
\begin{array}{ll}
M \longrightarrow M+1 & \text { rate: } V_{\max } \frac{P^{n}}{\frac{k_{1}^{n}}{d o x^{n}}+P^{n}}+b \\
M \longrightarrow M-1 & \text { rate: } \delta_{m} M \\
P \longrightarrow P+1 & \text { rate: } \mu M \\
P \longrightarrow P-1 & \text { rate: } \delta_{P} P
\end{array}
$$

Where $M, P$ and dox stand for mRNA, protein and doxycycline, respectively. $V_{\max }$ and $b$ define the maximal and minimal transcription rate, respectively. $k_{1} / d o x$ is the dissociation constant $\left(K_{d}\right)$ which defines the binding affinity of the TF to DNA. $n$ is the Hill coefficient, which indicates how cooperative is the binding of the TF. $\delta_{m}$ and $\delta_{p}$ are the degradation rates of the mRNA and protein, respectively. $\mu$ is the translation rate.

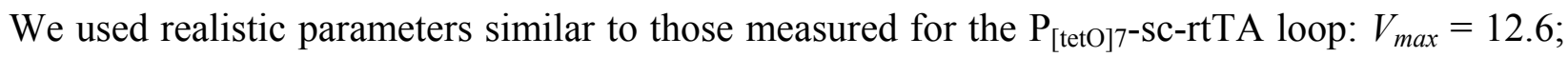
$k_{l}=6988.67 ; \delta_{m}=0.2291 ; \delta_{p}=0.0095 ; \mu=4.836$. All time units are expressed in minutes. The values for $b$ and $n$ are indicated in the figures.

The simulation was performed with the Gillespie stochastic simulation algorithm. The simulations were preceded by a pre-run to mimic initial condition with either the low or high expression state. A transition was detected whenever a trajectory passed the value defined by the geometric mean of the low and high expression states at the doxycycline concentration that is the geometric mean of the doxycycline concentrations at the two bistable boundaries.

\section{Determination of the bistability boundaries based on the open-loop functions}

The following open-loop functions $f_{\text {OLM }}$ were used: 
$f_{\text {OLM }}(\omega, d o x)=V_{\max } \frac{[g(\omega)]^{n_{1}}}{\frac{k_{1}}{d o x^{n_{2}}}+[g(\omega)]^{n_{1}}}+b$

For monomeric circuit $\left(\mathrm{P}_{[\text {tetO }] 7-S c-r t T A}\right) g(\omega)=\omega$, and for the dimeric circuit $\left(\mathrm{P}_{[\text {tetO }] 7-r t T A}\right)$ a dimerization term was used: $g(\omega)=\omega+0.5\left(\kappa-\sqrt{4 \omega \kappa+\kappa^{2}}\right)$. The parameter values for the dimeric circuit are: $V_{\max }=74.48 ; k_{1}=0.036 ; \kappa=719.7 ; n_{1}=1.46 ; n_{2}=1.32 ; b=0.78$. The parameter values for the monomeric circuit are: $V_{\max }=54.6 ; k_{1}=8.71 ; n_{1}=1.94 ; n_{2}=1.9 ; b=$ $0.78^{14}$.

To find the steady-state values for the feedback loops, the open-loop was reclosed so that the roots of the equation, $f_{O L M}(\omega, d o x)=s \omega$, were determined. $S$ is the scaling factor correcting the difference of expression between the mRNA of the input and output and its values is 1.4 and 2.29 for the dimeric and monomeric $\mathrm{TF}$, respectively.

The extremal value of the relative basal expression for the bistable domain as the function of the Hill-coefficient

For a family of one-gene loops mediated by a monomeric TF with a specified promoter response, $r(x)$, the closed loop system is described by $b+V_{\max } r(x)-\delta_{P} x=0$ at equilibrium. It can be opened to $x=f(\omega)=\delta_{P}^{-1}\left(b+V_{\max } r(\omega)\right)$. In the analysis below, $r(x)$ is the Hill function $h\left(x, K_{d}, n\right)$.

This degree is quantified by the logarithmic derivative, hence termed the logarithmic sensitivity $(S)$. If $S$ is larger than one, the reaction response is ultrasensitive. The logarithmic sensitivity of the open-loop function $f(\omega)$ is $S_{\omega}^{f}=\partial \ln f(\omega) / \partial \ln \omega$.

Due to the saturation of the open-loop function, $S_{\omega}^{f}$ approaches zero at large values of the input, $\omega$. For non-zero basal production rate, $b, S_{\omega}^{f}$ approaches zero as $\omega \rightarrow 0$. Thus, $S_{\omega}^{f}$ has a maximum point, whose value drops to one when $b / V_{\max }$ reaches a critical value: bistability is lost when $S_{\omega}^{f}=1$ and $\partial_{\omega} S_{\omega}^{f}=0$. These conditions result in $\partial_{\omega \omega} f(\omega) \cdot(\omega / f(\omega))=0$. At equilibrium, $\omega=f(\omega)$; consequently, $\partial_{\omega \omega} f(\omega)=0$ signifying that $\omega$ is an inflection point for $f(\omega)$. This is equivalent with the conditions for the cusp bifurcation if the system is at equilibrium.

The critical value of $b / V_{\max }$ can be expressed where bistability vanishes as

$\left(\frac{b}{V_{\max }}\right)_{\text {cusp }}=\left.\left(\mathrm{S}_{\omega}^{f}-1\right) h(\omega)\right|_{\omega=\omega_{\mathrm{inf}}}=\frac{(n-1)^{2}}{4 n}$

The general form of promoter response for dual positive-negative feedback loop is $V_{\max } h\left(x, K_{1}, n\right)\left(1-h\left(x, K_{2}, m\right)\right)+\mathrm{b}$. Thus, its open-loop function is 
$f(\omega)=\delta_{P}^{-1}\left(V_{\max } \frac{\omega^{n}}{K_{1}^{n}+\omega^{n}} \frac{K_{2}^{m}}{K_{2}^{m}+\omega^{m}}+b\right)$

Assuming $m=n$ and $K_{l}=K_{2}$, leads to a response function, for which an analytical solution for the critical point can be obtained.

$$
\left(\frac{b}{V_{\max }}\right)_{\text {cusp }}=\frac{2}{27 n^{2}}+\frac{\left(3 n^{2}+1\right)\left(-3+\sqrt{1+3 n^{2}}\right)}{54 n^{2}}
$$

\section{Acknowledgments}

We thank Farzaneh Maleki for help in the simulation of transition rates. Aurelio de los Reyes V and Imre Májer for the help in the derivations. This work was supported by grants from the Swiss National Foundation and the StoNets RTD from SystemsX. C.H. was a Long-Term Fellow of the Human Frontier Science Program.

\section{Author contributions}

A.B. designed the project. V.J. and C.H. performed the experiments and data analysis. A.B., C.H. and V.J. wrote the paper.

\section{References}

1. L. K. Nguyen, B. N. Kholodenko and A. von Kriegsheim, Small GTPases, 2016, 1-6.

2. Q. B. He and Z. R. Liu, Curr Bioinform, 2015, 10, 48-58.

3. P. Guerrero, H. M. Byrne, P. K. Maini and T. Alarcon, J Math Biol, 2016, 72, 123-156.

4. J. Narula, M. Fujita and O. A. Igoshin, Curr Opin Microbiol, 2016, 34, 38-46.

5. A. V. Zaytsev, D. Segura-Pena, M. Godzi, A. Calderon, E. R. Ballister, R. Stamatov, A. M. Mayo, L. Peterson, B. E. Black, F. I. Ataullakhanov, M. A. Lampson and E. L. Grishchuk, Elife, 2016, 5, e10644.

6. D. Kim, O. Rath, W. Kolch and K. H. Cho, Oncogene, 2007, 26, 4571-4579.

7. G. Charvin, C. Oikonomou, E. D. Siggia and F. R. Cross, PLoS biology, 2010, 8, e1000284.

8. X. J. Tian, H. Zhang and J. Xing, Biophys J, 2013, 105, 1079-1089.

9. D. Siegal-Gaskins, E. Franco, T. Zhou and R. M. Murray, J R Soc Interface, 2015, 12, 20150288.

10. J. E. Ferrell, Jr. and S. H. Ha, Trends Biochem Sci, 2014, 39, 612-618.

11. R. Hermsen, D. W. Erickson and T. Hwa, PLoS Comput Biol, 2011, 7, e1002265.

12. I. Majer, A. Hajihosseini and A. Becskei, Phys Biol, 2015, 12, 066011.

13. I. J. Roney, A. D. Rudner, J. F. Couture and M. Kaern, Sci Rep, 2016, 6, 27697.

14. C. Hsu, V. Jaquet, F. Maleki and A. Becskei, J Mol Biol, 2016, 428, 4115-4128.

15. J. Vinuelas, G. Kaneko, A. Coulon, E. Vallin, V. Morin, C. Mejia-Pous, J. J. Kupiec, G. Beslon and O. Gandrillon, BMC Biol, 2013, 11, 15.

16. R. Arbel-Goren, A. Tal and J. Stavans, Wiley Interdiscip Rev RNA, 2014, 5, 197-207.

17. M. Scott, T. Hwa and B. Ingalls, Proc Natl Acad Sci U S A, 2007, 104, 7402-7407.

18. F. Maleki and A. Becskei, J Theor Biol, 2017, /dx.doi.org/10.1016/j.jtbi.2016.12.012.

19. I. C. MacDonald and T. L. Deans, Adv Drug Deliv Rev, 2016, 105, 20-34.

20. L. Z. Wang, F. Wu, K. Flores, Y. C. Lai and X. Wang, Integr Biol (Camb), 2016, 8, 394-408. 
21. T. L. To and N. Maheshri, Science, 2010, 327, 1142-1145.

22. T. May, L. Eccleston, S. Herrmann, H. Hauser, J. Goncalves and D. Wirth, PLoS One, 2008, 3, e2372.

23. N. T. Ingolia and A. W. Murray, Current biology: $C B, 2007,17,668-677$.

24. D. Del Vecchio, A. J. Dy and Y. Qian, J R Soc Interface, 2016, 13.

25. O. P. Tabbaa and C. Jayaprakash, Phys Biol, 2014, 11, 046004.

26. K. Axelrod, A. Sanchez and J. Gore, Elife, 2015, 4.

27. C. Hsu, V. Jaquet, M. Gencoglu and A. Becskei, Cell Rep, 2016, 16, 1204-1210.

28. G. Belli, E. Gari, L. Piedrafita, M. Aldea and E. Herrero, Nucleic Acids Res, 1998, 26, 942-947.

29. J. Hu, K. R. Qin, C. Xiang and T. H. Lee, Bull Math Biol, 2012, 74, 1727-1753.

30. C. Song, H. Phenix, V. Abedi, M. Scott, B. P. Ingalls, M. Kaern and T. J. Perkins, PLoS Comput Biol, 2010, 6, e1000699.

31. C. Hsu, S. Scherrer, A. Buetti-Dinh, P. Ratna, J. Pizzolato, V. Jaquet and A. Becskei, Nat Commun, $2012,3,682$. 


\section{Figure legends}

Fig. 1. Relation between bifurcations and transition rates

(A) The stable (full) and unstable (dashed curves) steady-state expression levels of a positive feedback loop with a monomeric TF. The bistability boundaries (double-headed arrows) are indicated for the different relative basal expression levels $\left(b / V_{\max }\right)$. The equation corresponds to the deterministic form (steady-state form of the ordinary differential equation) of the reactions shown in equation (1).

(B) The open-loop system is obtained by breaking the loop and the ultrasensitivity can be characterized. When the basal expression is increased, the dynamic range of the promoter and ultrasensitivity is reduced (bottom left panel). Conversely, when the Hill coefficient is increased the ultrasensitivity of the open-loop function is increased (bottom right panel).

(C) Stochastic simulations were used to predict the transition rates from high to low expression state (triangle pointing down) and from the low to the high state (triangle pointing up). The bistable range corresponds to that shown in (A).

(D) Stability diagram displays the bistable region (delimited by the black lines) as a function of the TF-DNA affinity and basal expression. The transition rates in reported in (C) are color coded. The purple circle stands for the cusp point, i.e. the point which delimits the extremal point of the relative basal expression where bistability is possible, $b / V_{\max }=0.125$. The dashed lines indicated the presumptive bistable region based on the threshold transition rate of $0.02 \mathrm{~h}^{-1}$.

Fig. 2. Measurement and interpolation of the transition rates

(A) Feedback loop design. The synthetic circuit was composed of a positive feedback construct, where TF activates its own expression and an additional construct to adjust the basal expression.

(B) The transition rates from OFF to ON (left panels) and from ON to OFF expression states (right panels) for the non-cooperative (upper panels) and cooperative (lower panels) tTA feedback loops are plotted as function of doxycycline concentration. The relative basal expression was adjusted by the estradiol concentration. At each basal expression, the OFF-to-ON and $\mathrm{ON}-$ to-OFF transition rates are fitted as function of doxycycline concentration by a linear regression and by a power regression $\left(a \cdot d o x^{b}\right)$, respectively. The transition rate threshold was set to $0.02 \mathrm{~h}^{-1}$ (horizontal gray lines). 
Fig. 3. Comparison of the bistability boundaries obtained by using the open-loop approach and the transition rates

(A,B) The threshold values from Fig. 2B and Fig. S3 are reported for the OFF-to-ON (triangles pointing up) and $\mathrm{ON}-$ to-OFF (triangles pointing down) transition rates. Their relative basal expressions were modulated by different estradiol concentrations indicated in Fig. $2 \mathrm{~B}$ and Fig. S3 where the lowest concentration corresponds to leftmost triangles. The full lines stand for the presumptive bistable boundaries which were obtained by linear regression of the base- 10 logarithm values of the transition rate threshold set to $0.02 \mathrm{~h}^{-1}$. The cusp point which is the extreme point of bistability is indicated by a purple circle. The presumptive bistable domains for the tTA feedback loops (A). The bistable boundaries mapped by the open-loop approach (dashed lines) and estimated with the transition rates (full lines) for the dimeric - cooperative (blue) and monomeric - cooperative (red) (sc-)-rtTA feedback loops (B).

(C,D) Predicted (contour diagram) and observed (triangles) transition rates (indicated by a color scale) for monomeric (C) and dimeric (D) cooperative feedback loops. The open-loop approach is used to map the bistable boundaries which are identical as the dashed lines in (B) (cyan-whitecyan lines). The predicted transition rates were obtained by stochastic simulation with parameters as described (see extended fitted noise model and fitted transient kinetics in reference ${ }^{14}$ ). Differences between predicted and observed transition rates can be seen by the distance in the color-scale. The band in the bottom left corner in (D) stands for the ON-to-OFF transitions.

Fig. 4. The effect of the ultrasensitivity of the open-loop function with respect to the system variable (input) or system parameter (doxycycline signal) on the extension of the bistable domain.

(A) Molecular reactions in the open loop. The input and output are mRNA molecules. (B,C) Open-loop function of the monomeric (red) and dimeric (blue) cooperative (sc-)rtTA circuit as function of input (B) or doxycycline (C) concentrations. (D-E) Prediction of the extension of the bistable region of $\mathrm{P}_{[\mathrm{tetO}] 7}$-rtTA loop by varying the Hill-coefficient with respect to the input (D) and with respect to the doxycycline $(\mathrm{E})$. The blue lines are identical to the blue dashed lines in Fig. 3B and the cyan lines in Fig. 3D.

Fig. 5. Bistability region as a function of the promoter dynamic range

(A) The presumptive bistable domains for the dimeric - non-cooperative (gray lines) and the dual positive-negative (cyan lines) feedback loops.

(B) Extremal values of the bistable domains (cusp points) based on the transition rates (full symbols) and open-loop function (empty symbols). The points are positioned along the function that links $\left(b / V_{\max }\right)_{\text {cusp }}$ to the Hill-coefficient, for simple positive feedback loops (full line, equation (3)) and for the dual positive-negative feedback loop (dashed line, equation (5)). 
(C) The bistable range of the inducer concentration (left panel) is the ratio of the inducer concentrations at the estimated bistability boundaries. It was determined at the lowest (endogenous) basal expression, which is variable in the different feedback loops, and at a higher but equal basal expression, interpolated for all feedback loops. The relative basal expression at the cusp point is shown in the right panel estimated by the threshold in the transition rates. 


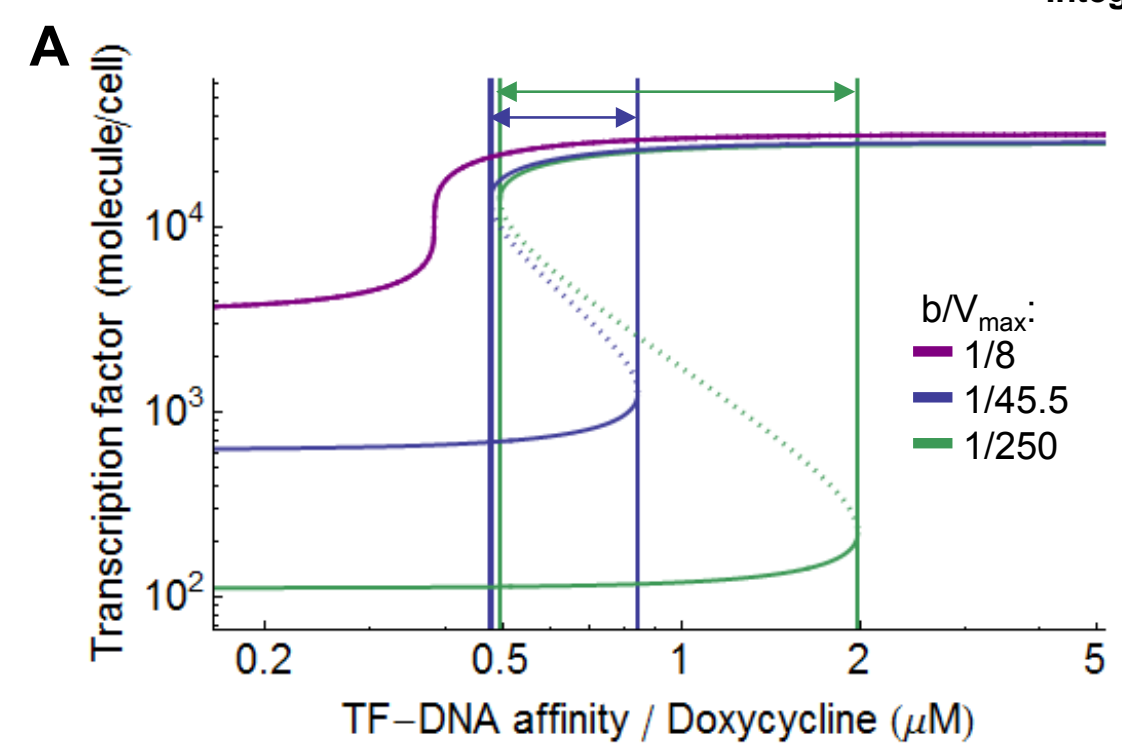

C

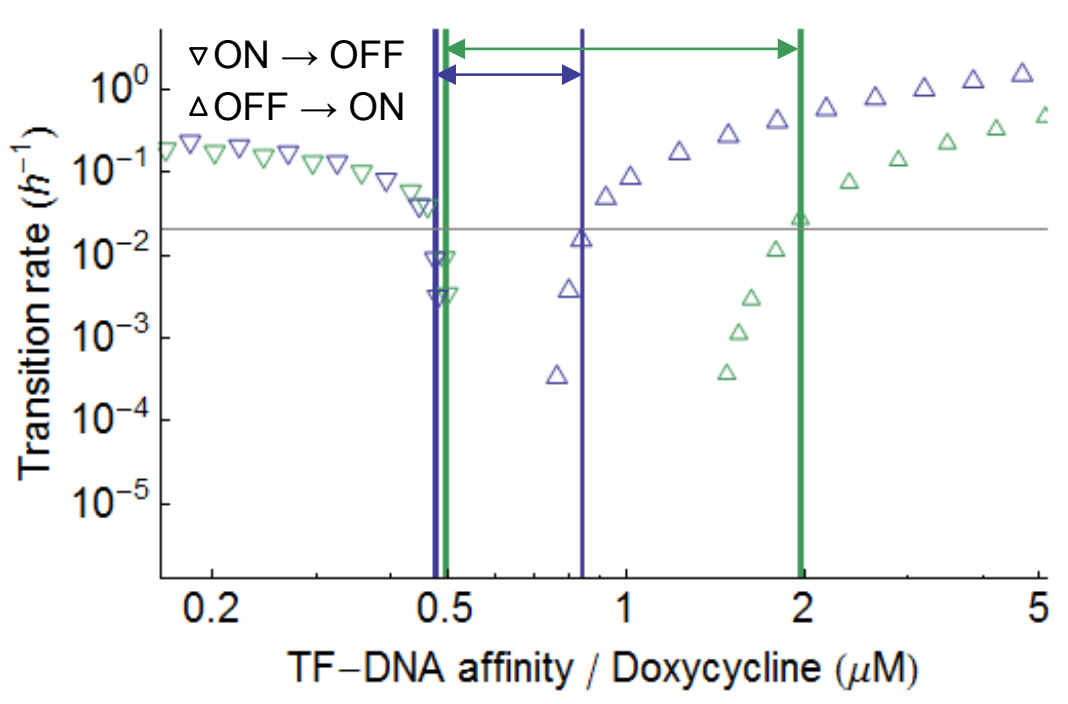

B
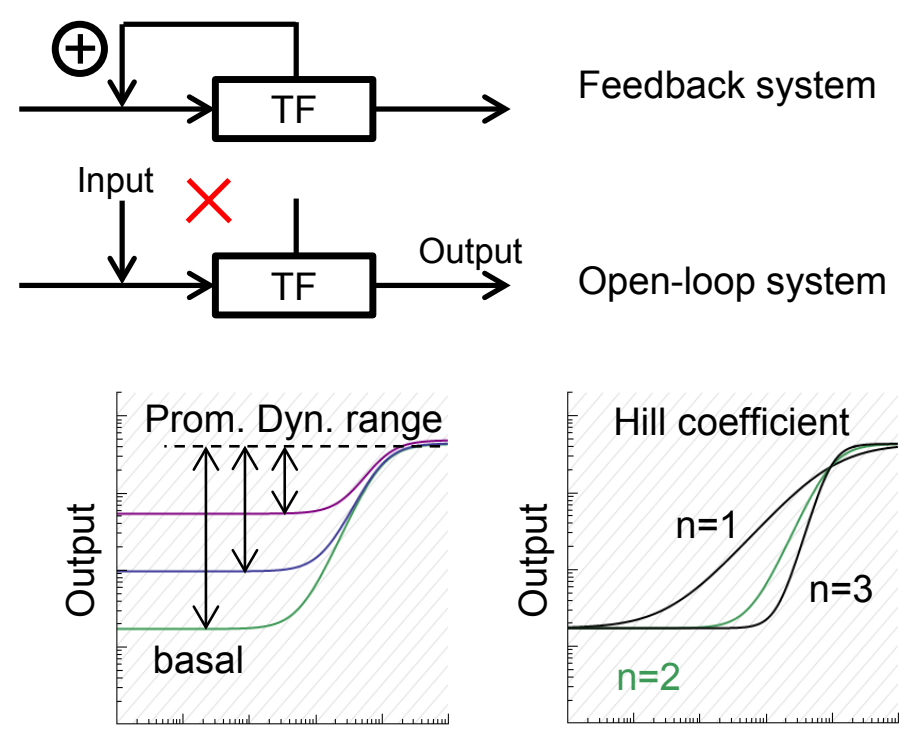

Input

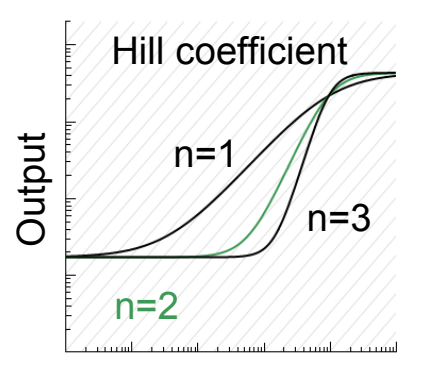

D

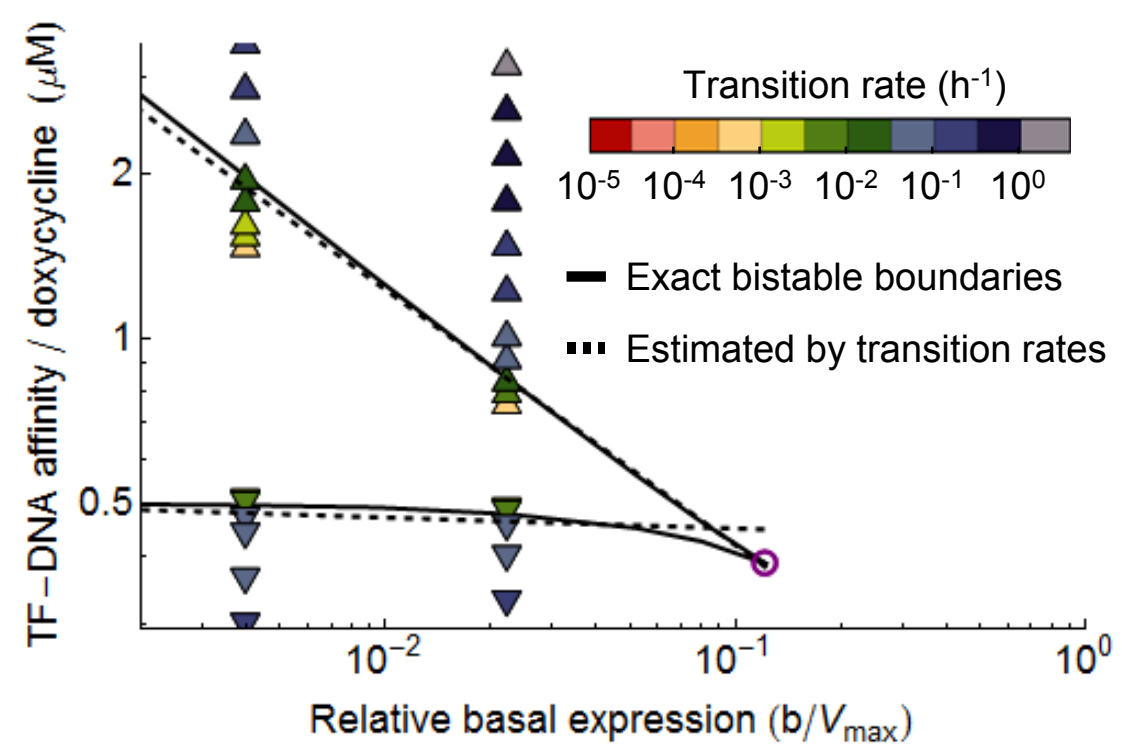

Figure 1 


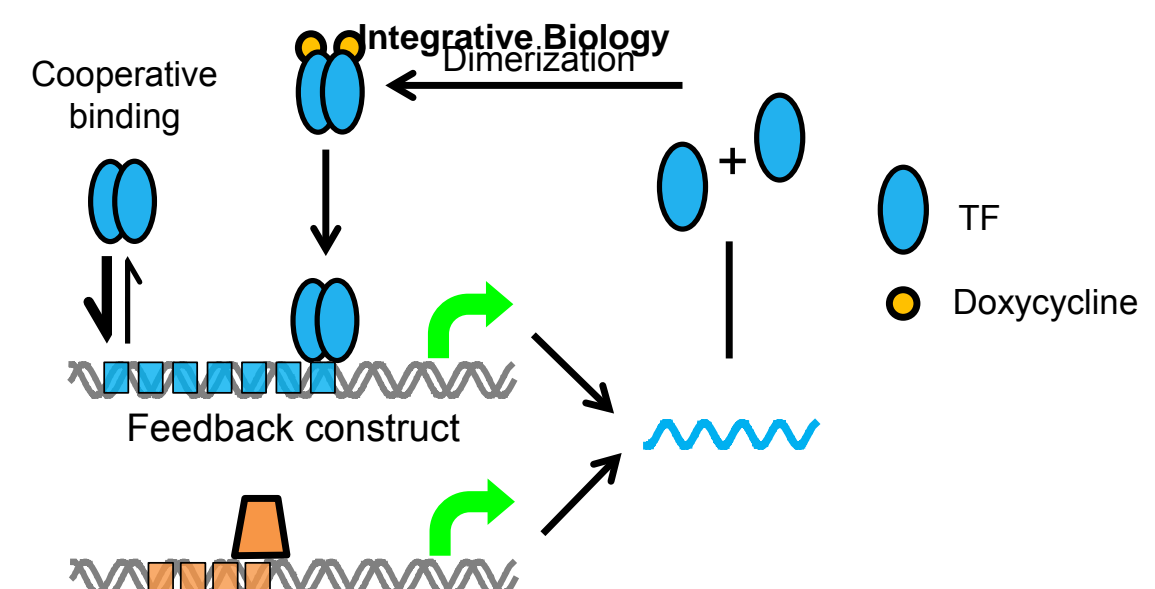

Basal adjustment construct

B
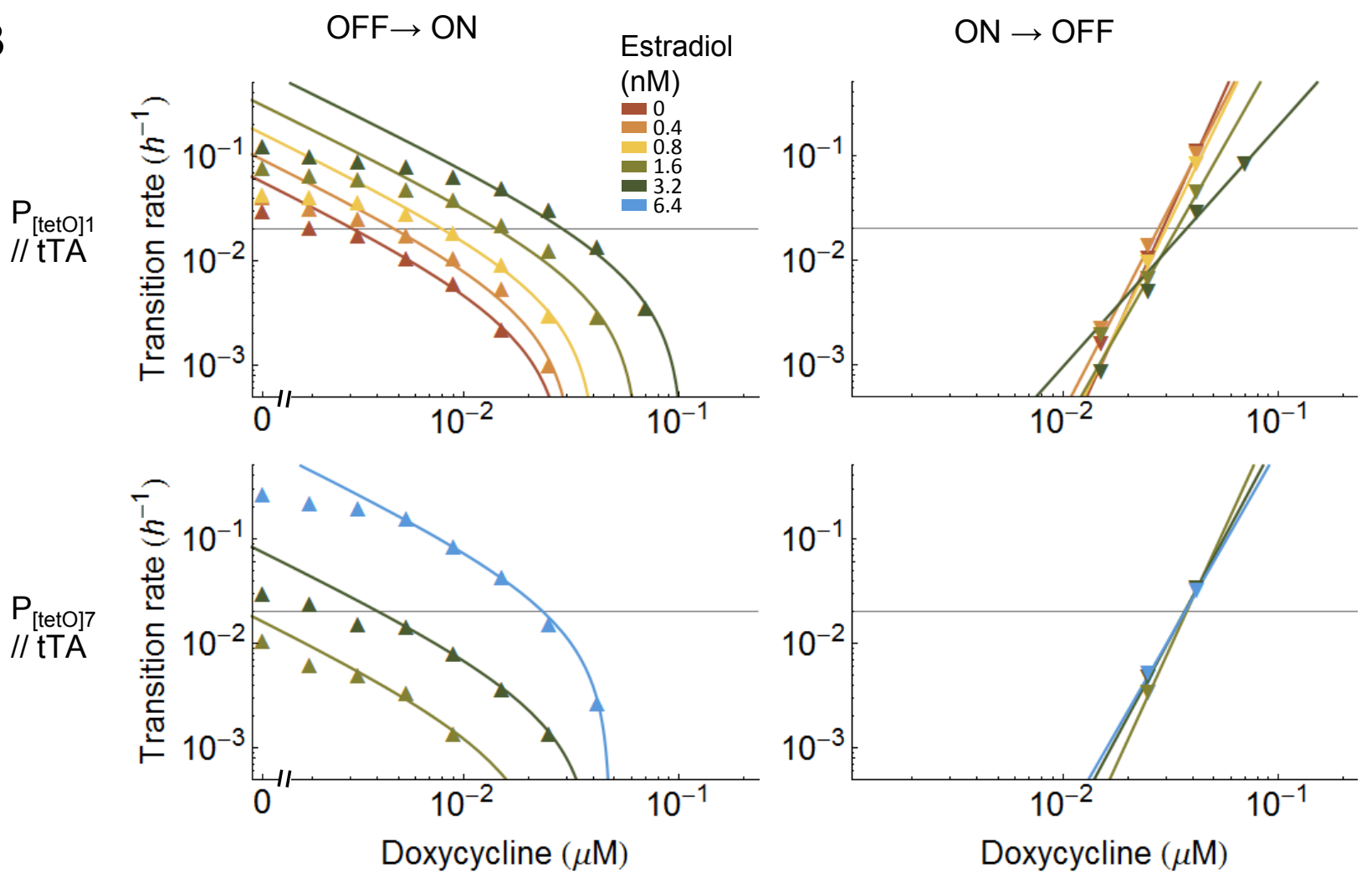

Figure 2 

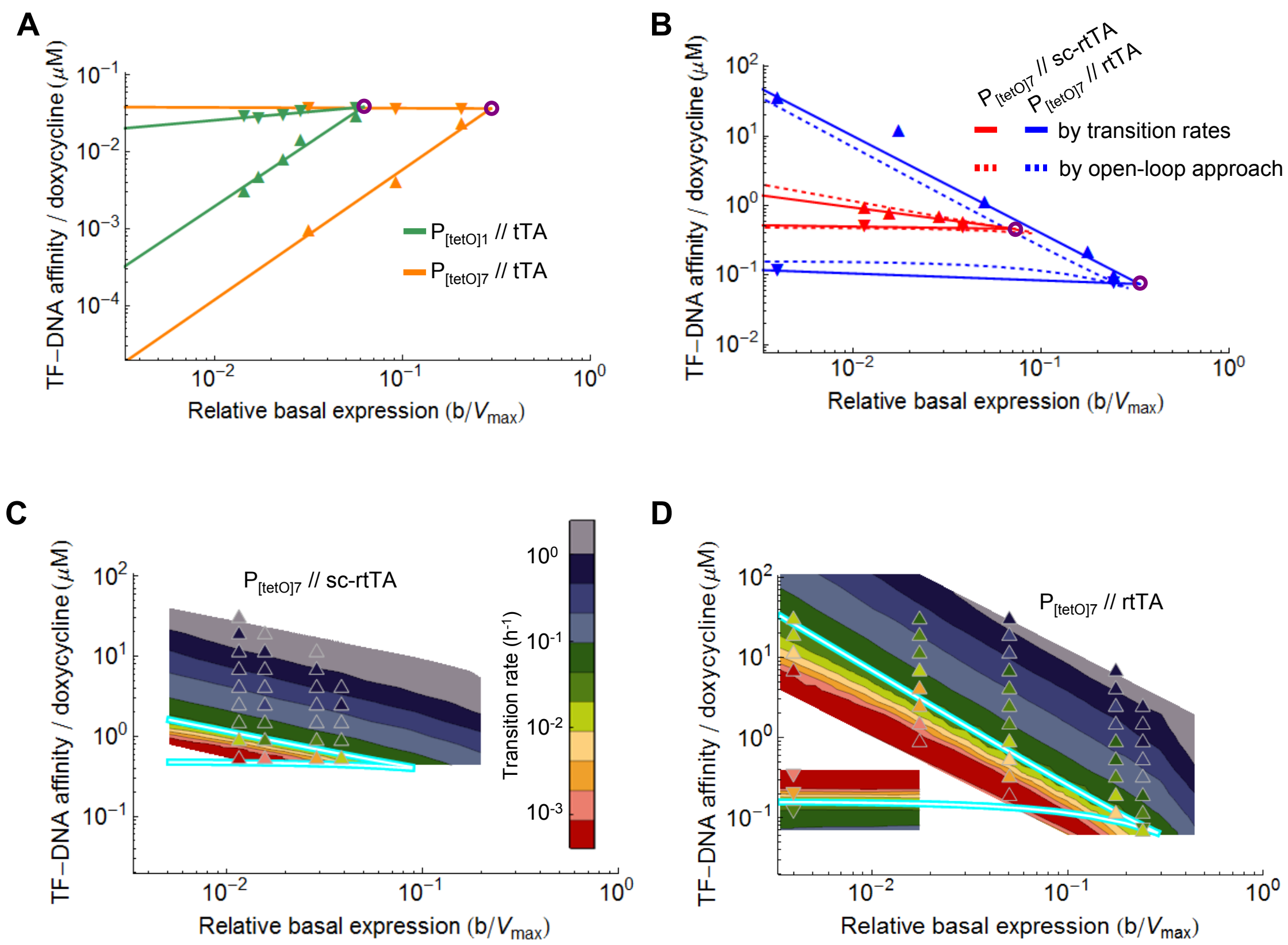

Figure 3 
A

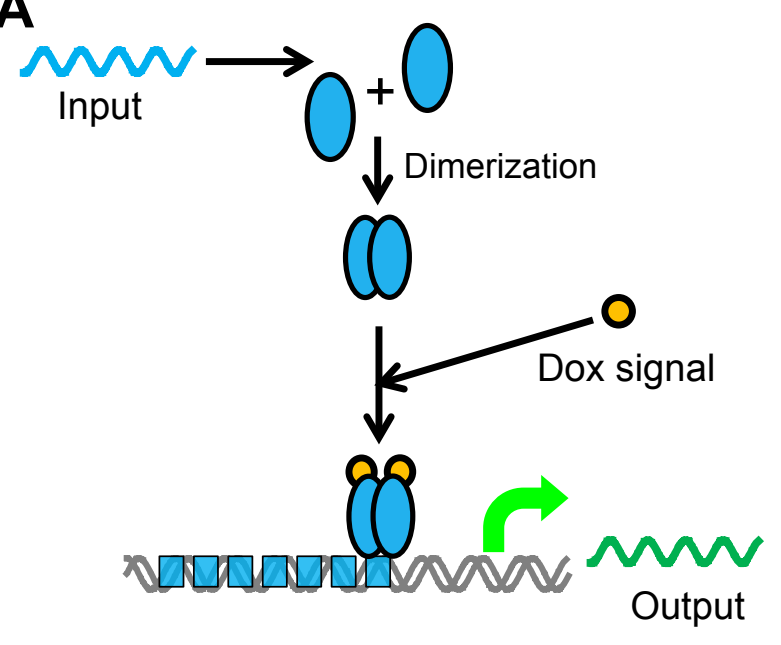

B

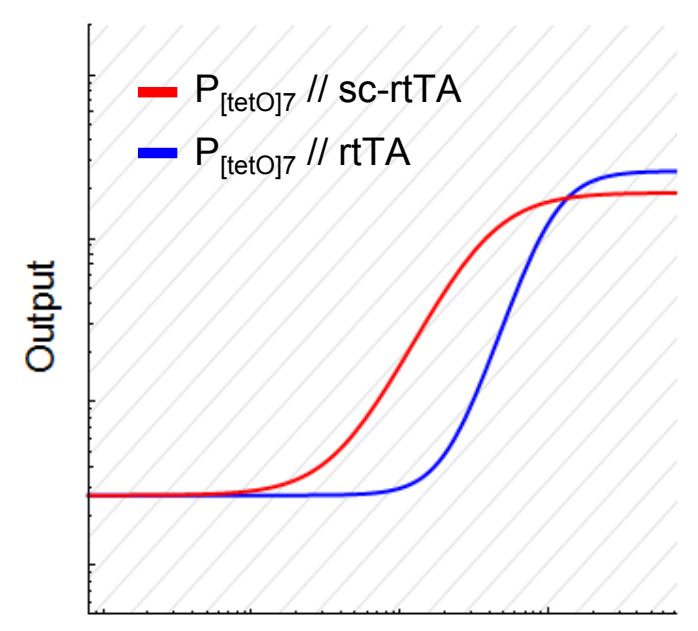

Input
C

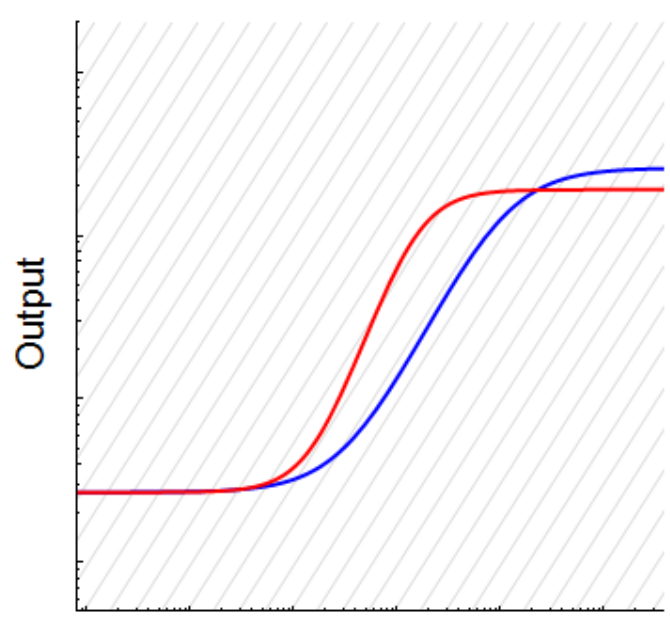

Dox signal
D

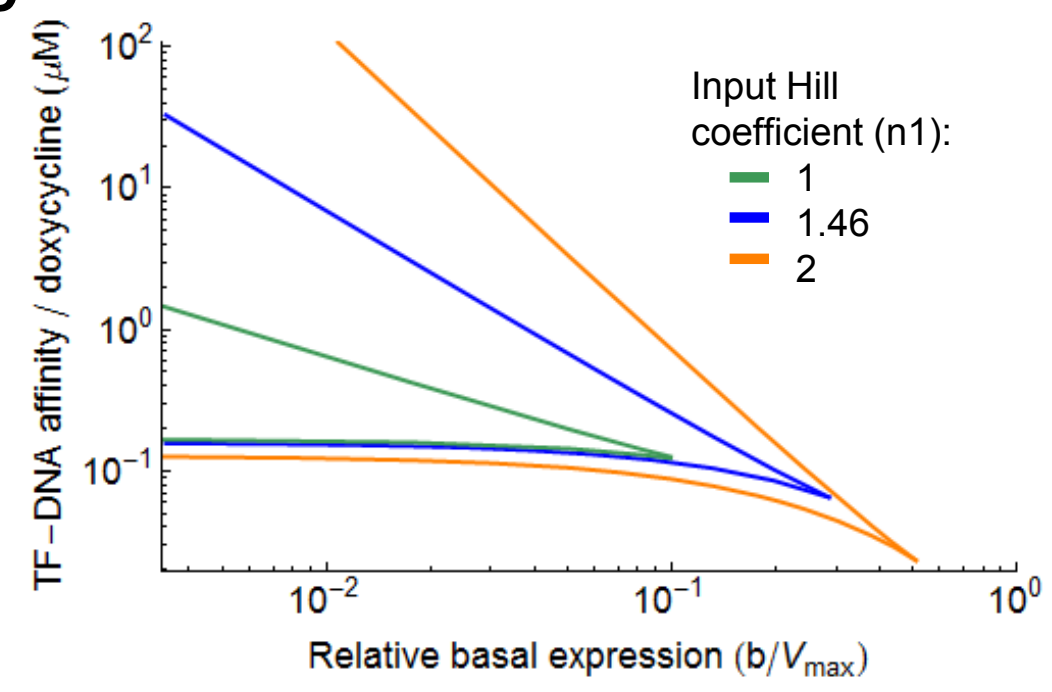

E

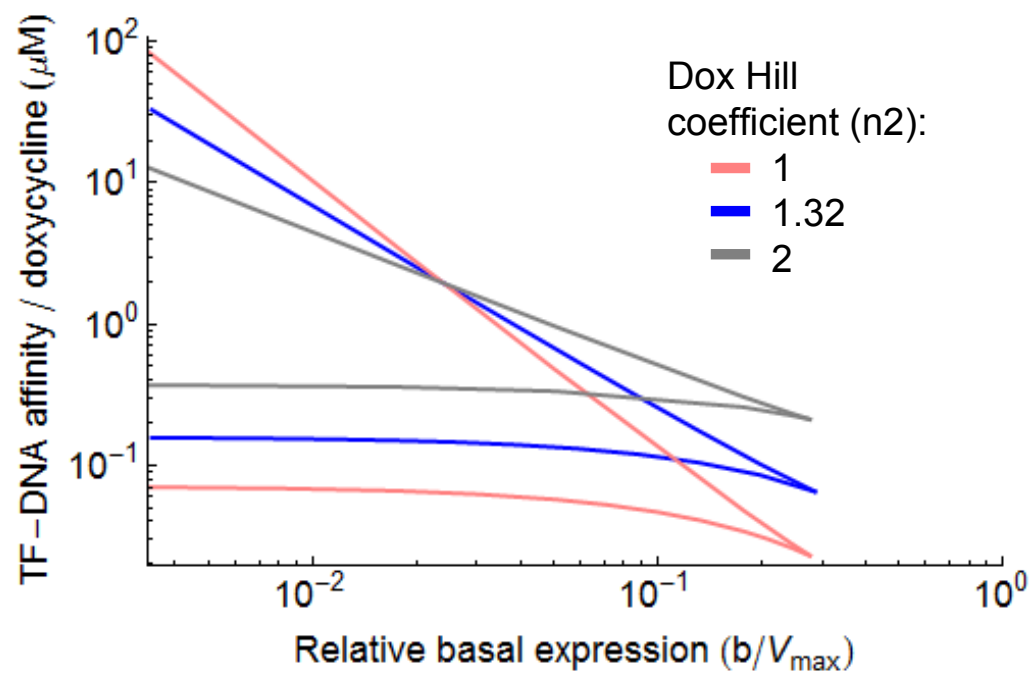

Figure 4 

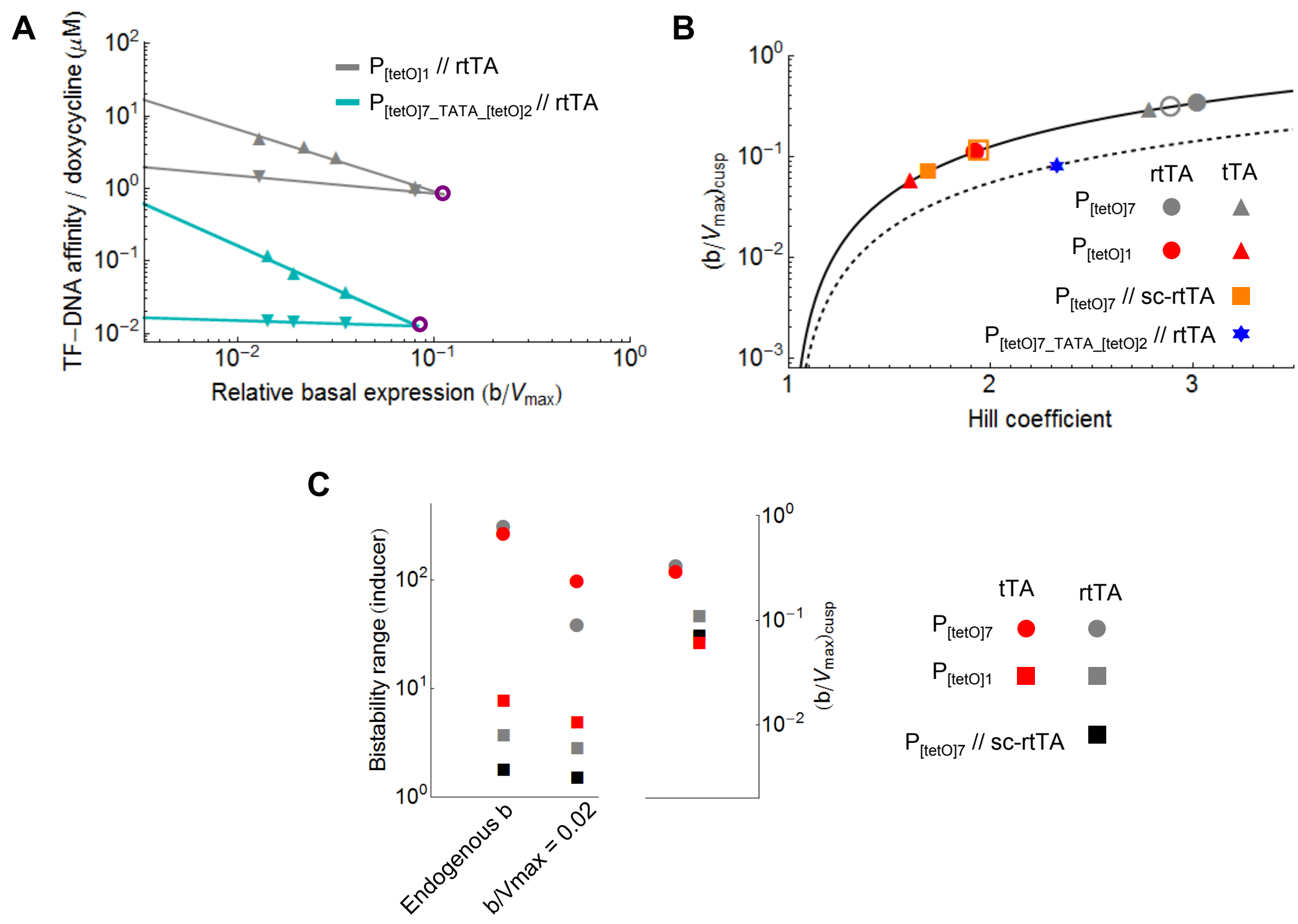

Figure 5 


\section{Page 25 of 56}

A

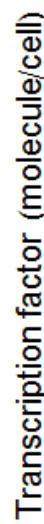

$n_{\mathrm{H}}=1.5$

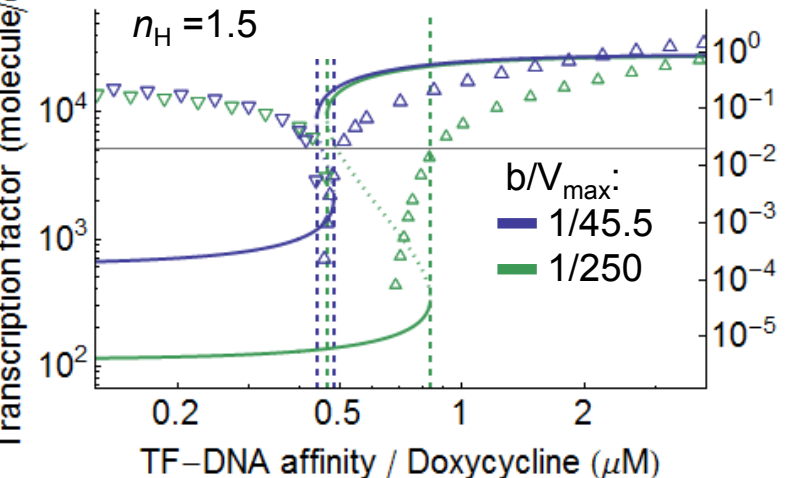

TF-DNA affinity / Doxycycline $(\mu \mathrm{M})$

\section{Integrative Biology}

B

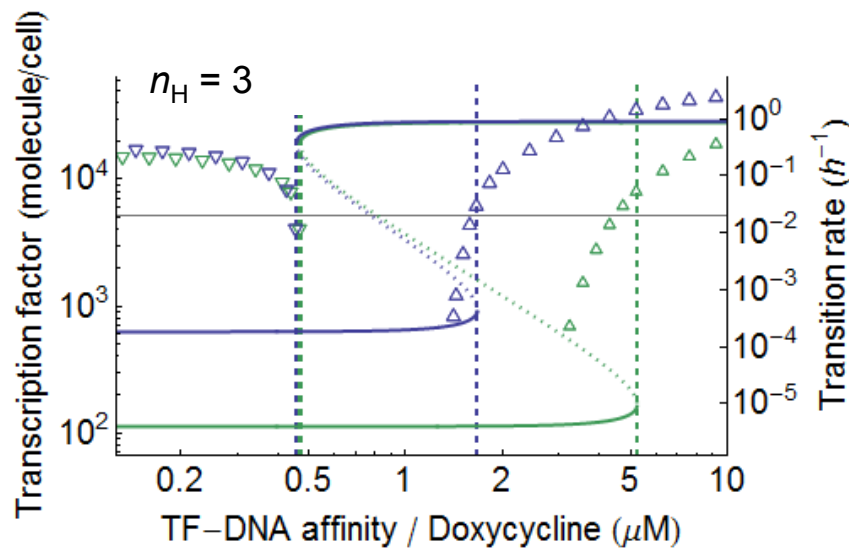

C

\begin{tabular}{|cc|ccc|ccc|}
\hline Vmax/b & $\begin{array}{c}\text { Hill coef. } \\
\left(n_{H}\right)\end{array}$ & \multicolumn{2}{|c|}{$\begin{array}{c}\text { Left boundary, } \\
\text { Doxycycline }(\mu \mathrm{M}) \\
\text { exact }\end{array}$} & estimated & \% error & \multicolumn{3}{|c|}{ Right boundary, } \\
Doxycycline $(\mu \mathrm{M})$ & \\
exact & estimated & \% error \\
\hline 250 & 1.5 & 0.4667 & 0.4492 & -3.8 & 0.8357 & 0.8524 & 2.0 \\
\hline 45.5 & 1.5 & 0.4406 & 0.4282 & -2.8 & 0.4842 & 0.5038 & 4.0 \\
\hline 250 & 2 & 0.4954 & 0.4629 & -6.6 & 1.9767 & 1.8851 & -4.6 \\
\hline 45.5 & 2 & 0.4775 & 0.4794 & 0.4 & 0.8432 & 0.8434 & 0.0 \\
\hline 250 & 3 & 0.4699 & 0.4628 & -1.5 & 5.2364 & 4.4320 & -15.4 \\
\hline 45.5 & 3 & 0.4571 & 0.4498 & -1.6 & 1.6674 & 1.6207 & -2.8 \\
\hline
\end{tabular}

Fig. S1 Accuracy of the approximation of the bistable boundaries by a threshold of transition rate at $0.02\left(\mathrm{~h}^{-1}\right)$ in feedback loops with weakly and strongly cooperative binding of the TF. (A,B) The same parameters were used as in Fig. 1A,C, except for the Hill coefficient $n_{\mathrm{H}}=1.5$ in (A) and 3 in (B). (C) The exact bifurcation points are compared with the estimated ones at threshold of transition rate $0.02\left(\mathrm{~h}^{-1}\right)$. The relative errors are calculated for each value of basal expression. 


\section{Integrative Biology}

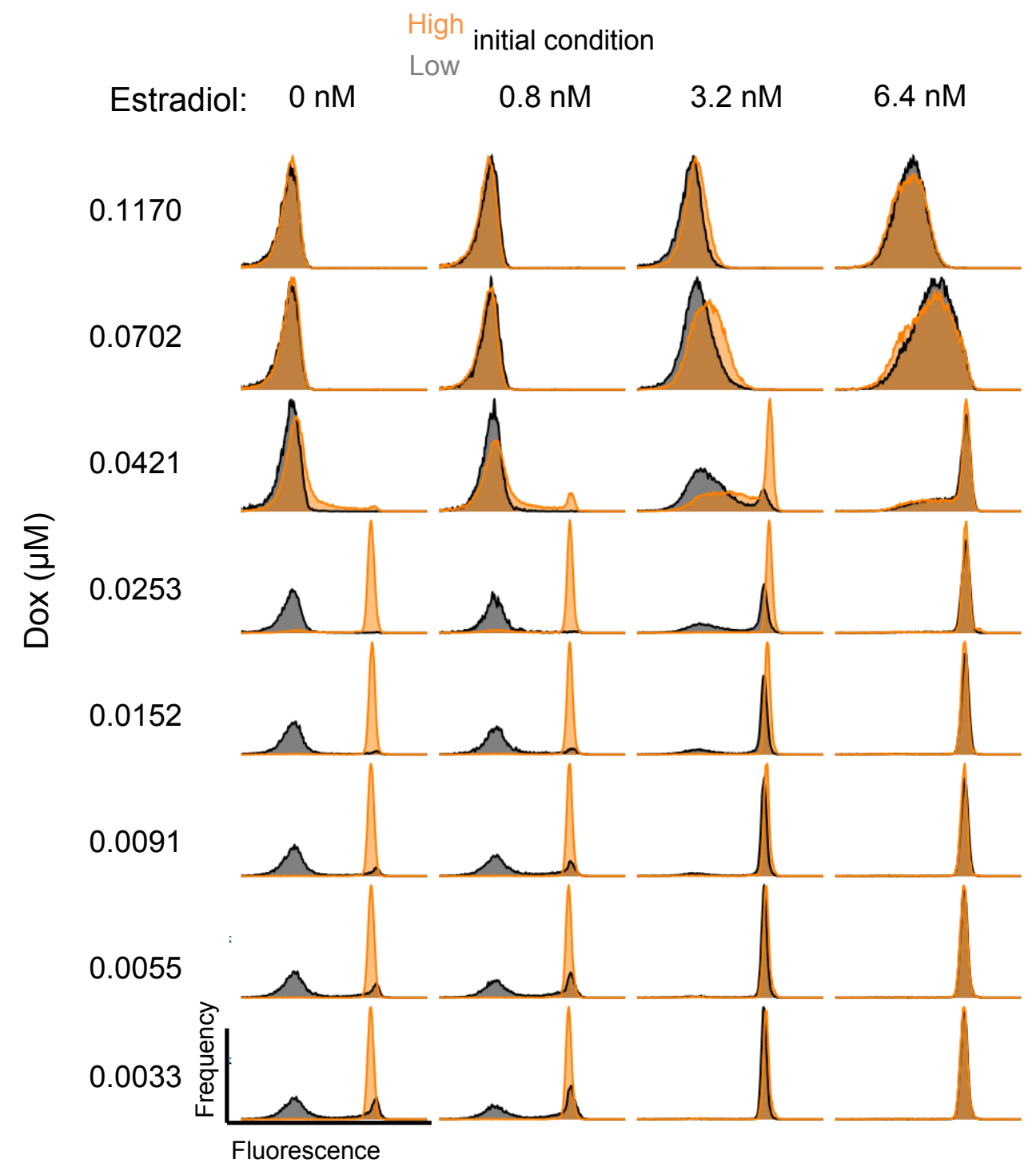

Fig. S2 Histograms of fluorescence distributions of the $\mathrm{P}_{[\text {tetO]1 }}$ - $\mathrm{tTA}$ feedback loop as doxcycycline and estradiol is varied The low and high initial conditions were set by incubating the cells in the absence or presence of galactose (at $0.5 \%$ ). Measurements were performed after transferring the cells from the above initial conditions into the media with the indicated doxycycline concentration and estradiol and were grown for an additional 24h. The tTA activity in the feedback loops in the cells was reported with GFP. This figure is related to Fig. 3A. 

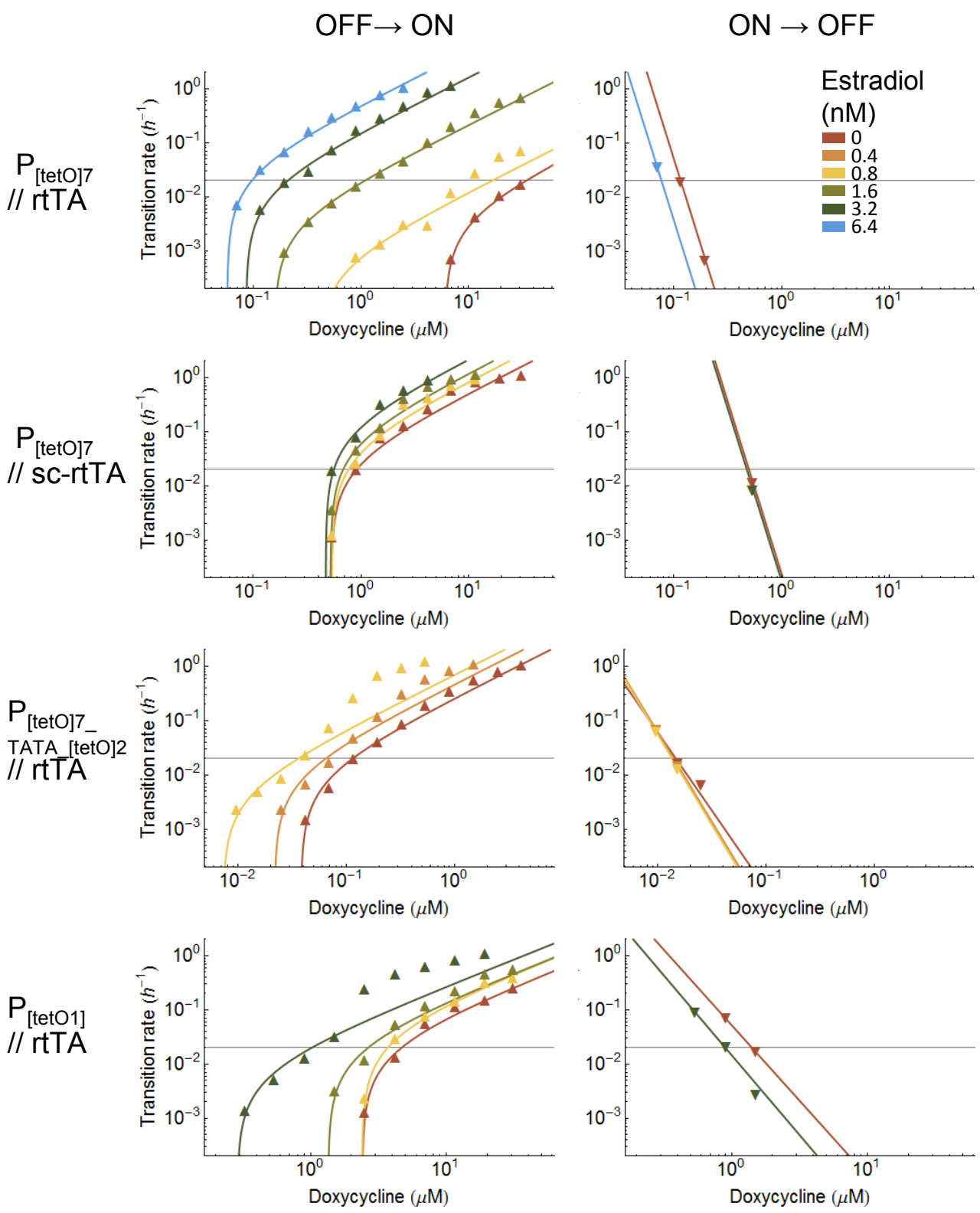

Fig. S3 Measured transition rates between the two expression states in feedback circuits. The transition rates from OFF to ON (left panels) and from ON to OFF expression states (right panels) for 4 different feedback loops were plotted as function of doxycycline concentration. The feedback expression range was adjusted by the estradiol concentration which controls the basal expression (see methods). At each feedback expression range, the OFF-to-ON and ON-to-OFF transition rates were fitted as function of doxycycline concentration by a linear regression and by a power regression, respectively. The threshold used in Fig. 3B and 5A (gray horizontal lines) was set at $0.02 \mathrm{~h}^{-1}$. 


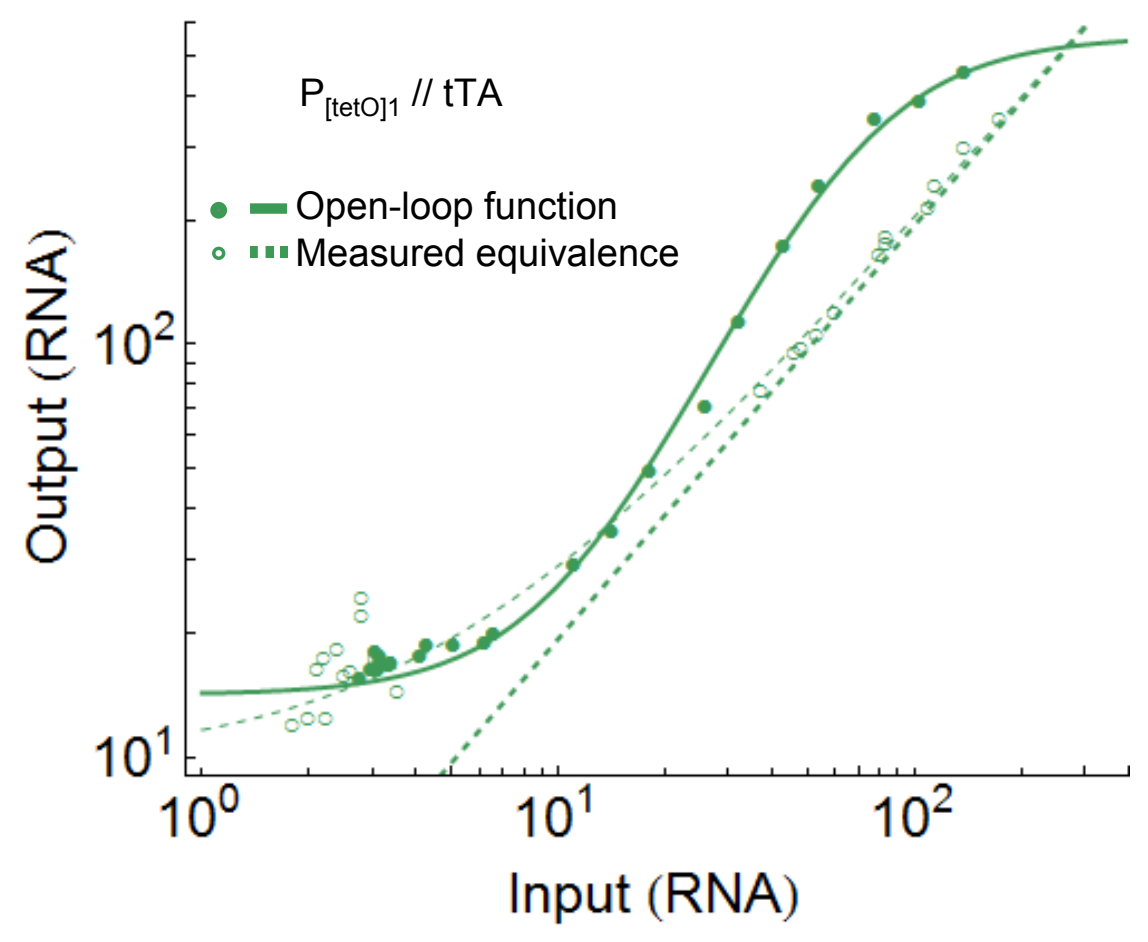

Fig. S4 Assessment of the ultrasensitivity due to the dimerization of tTA in open-loop construct. The input and output RNA was measured by varying the activity of the GAL promoter with estradiol at $0.012 \mu \mathrm{M}$ doxycycline in $\mathrm{P}_{[\mathrm{tetO}] 1}$-tTA Input/Output cell. The data for the open-loop function were fitted to Hill function with basal term and the measured equivalence to linear function, $s$ input $+b$, where $\mathrm{b}$ is the basal expression of the output. The fitted values for open-loop function were: $\mathrm{V}_{\max }=541.30, \mathrm{~b}=14.19, \mathrm{~K}_{\mathrm{d}}=66.57, \mathrm{n}=2.00$. The thick dashed lines for the measured equivalence are the linear function without $b$. 


\section{Supplementary Tables}

Table S1. Yeast strains.

\begin{tabular}{|c|c|c|c|c|c|}
\hline \multirow{3}{*}{$\begin{array}{l}\text { Diploid } \\
\text { Strain }\end{array}$} & \multirow{3}{*}{$\begin{array}{c}\begin{array}{c}\text { Haploid } \\
\text { parents }\end{array} \\
\text { A } \\
\text { alpha }\end{array}$} & \multicolumn{3}{|c|}{ Integration locus (plasmid) } & \multirow[t]{3}{*}{ Function } \\
\hline & & \multirow{2}{*}{ ade $2: \because \boldsymbol{A D E}$ _ } & \multirow{2}{*}{ ura $3:: \boldsymbol{U R} \boldsymbol{A} 3_{-}$} & \multirow{2}{*}{ his $3:: \boldsymbol{H I S}_{-}$} & \\
\hline & & & & & \\
\hline \multirow{2}{*}{ Yvj87.2* } & Yvj79.2 & & $\begin{array}{l}\text { P_[tetO]7-CYClc } \mid \text { SL_5[AT]1 | } \\
\text { rtTA (pCH068) }\end{array}$ & $\begin{array}{ll}\text { P_MRP7 } & \text { GEV } \\
(\mathrm{pPR} 1) & \end{array}$ & \multirow{2}{*}{ Feedback } \\
\hline & Yvj70.1 & $\begin{array}{l}\mathrm{P} \_ \text {GALIUAS-CYC1c | } \\
\mathrm{SL} \text { 5[AT]1 |rtTA (pVJ46) }\end{array}$ & $\begin{array}{l}\text { P_[tetO]2-CYClc } \mid y E G F P \\
\text { (pABG10) }\end{array}$ & & \\
\hline \multirow{2}{*}{ Yvj99* } & Yvj89.1 & & $\begin{array}{l}\mathrm{P} \_\left[\text {tetO]1-CYClc }\left|\mathrm{SL} \_5[\mathrm{AT}] 2\right|\right. \\
\mathrm{rtTA}(\mathrm{pVJ} 42)\end{array}$ & \begin{tabular}{l|l} 
P_MRP7 & GEV \\
$($ pPR1)
\end{tabular} & \multirow{2}{*}{ Feedback } \\
\hline & Yvj91.6 & $\begin{array}{l}\mathrm{P} \text { P_GALIUAS-CYClc | } \\
\text { SL 5[AT]2 IrtTA (pCH094) }\end{array}$ & $\begin{array}{l}\text { P_[tetO]2-CYClc } \mid y E G F P \\
(\text { pABG10) }\end{array}$ & & \\
\hline \multirow{2}{*}{ Yvj151.3* } & Yvj150.3 & $\begin{array}{l}\mathrm{P}[\text { [tetO]7-TATA-[tetO]2 } \\
C Y C 1 \mathrm{c} \mid \mathrm{rtTA}(\mathrm{pMG} 01)\end{array}$ & & $\begin{array}{l}\text { P_MRP7 } \\
(\mathrm{pPR} 1)\end{array}$ & \multirow{2}{*}{ Feedback } \\
\hline & Ych178.2 & $\begin{array}{l}\text { P_GAL1UAS-CYClc } \mid \mathrm{rtTA} \\
(\mathrm{pCH} 099)\end{array}$ & $\begin{array}{l}\text { P_[tetO]1-CYClc } \mid y E G F P \\
(\text { pCH001) }\end{array}$ & & \\
\hline \multirow[b]{2}{*}{$Y \operatorname{ch} 260.2^{*}$} & Yvj80.1 & & $\begin{array}{l}\text { P_[tetO]7-CYClc }|\mathrm{SL} 55[\mathrm{AT}] 3| \\
\text { sc-rtTA (pCH91) }\end{array}$ & $\begin{array}{l}\text { P_MRP7 | GEV } \\
(\mathrm{pPR} 1)\end{array}$ & \multirow[b]{2}{*}{ Feedback } \\
\hline & $Y \operatorname{ch} 250.2$ & $\begin{array}{l}\mathrm{P} \text { P_GALIUAS-CYClc | } \\
\mathrm{SL} 5 \text { [AT]3 | sc- } \\
\mathrm{rtTA}(\mathrm{pCH} 102)\end{array}$ & $\begin{array}{l}\text { P_[tetO]2-CYClc } \mid y E G F P \\
(\text { pABG10) }\end{array}$ & & \\
\hline \multirow{2}{*}{ Yvj139.1 } & Yvj134. 1 & & $\begin{array}{l}\text { P_[tetO]1-CYClc } \mid \text { SL5[AT]1- I } \\
\text { tTA (pCH077) }\end{array}$ & $\begin{array}{l}\text { P_MRP7 } \\
(\mathrm{pPR} 1)\end{array}$ & \multirow{2}{*}{ Feedback } \\
\hline & Ych150.7 & $\begin{array}{l}\mathrm{P}=\text { GALIUAS-CYClc | } \\
\text { SL 5[AT]1 ItTA (pCH085) }\end{array}$ & $\begin{array}{l}\text { P_[tetO]2-CYClc } \mid y E G F P \\
\text { (pABG10) }\end{array}$ & & \\
\hline \multirow{2}{*}{ Yvj138.48 } & Yvj133.4 & & $\begin{array}{l}\text { P_[tetO]7-CYClc } \mid \text { SL_6[AT]0 | } \\
\text { tTA (pCH061) }\end{array}$ & $\begin{array}{l}\text { P_MRP7 } \\
(\mathrm{pPR} 1)\end{array}$ & \multirow{2}{*}{ Feedback } \\
\hline & Yvj135.8 & $\begin{array}{l}\text { P_GAL1UAS-CYC1c l } \\
\text { SL 6[AT]0 |tTA (pCH062) }\end{array}$ & $\begin{array}{l}\text { P_[tetO]2-CYClc } \mid y E G F P \\
\text { (pABG10) }\end{array}$ & & \\
\hline \multirow{2}{*}{ Yvj143 } & Yvj40.3 & & & $\begin{array}{l}\text { P_MRP7 } \\
(\mathrm{pPR} 1)\end{array}$ & \multirow{2}{*}{$\mathrm{I} / \mathrm{O}$} \\
\hline & Ych151.5 & $\begin{array}{l}\mathrm{P} \_ \text {GALIUAS-CYClc | } \\
\mathrm{SL} \text { 5[AT]1 ItTA (pCH085) }\end{array}$ & $\begin{array}{l}\text { P_[tetO]1-CYC1c I SL_5[AT]1 } \\
\text { | tTA } \operatorname{tT}(45 / 45):: Y F P(p C H 066)\end{array}$ & & \\
\hline \multirow{2}{*}{ Yvj142 } & Yvj40.3 & & & $\begin{array}{l}\text { P_MRP7 | GEV } \\
\text { (pPR1) }\end{array}$ & \multirow{2}{*}{$\mathrm{I} / \mathrm{O}$} \\
\hline & Ych107.1 & $\begin{array}{l}\text { P_GAL1UAS-CYC1c l } \\
\text { SL 6[AT]0 |tTA (pCH062) }\end{array}$ & $\begin{array}{l}\text { P_[tetO]7-CYC1c I SL_6[AT]0 } \\
\mid \text { rtTAA(45/45)::YFP (pCH058) }\end{array}$ & & \\
\hline
\end{tabular}

${ }^{*}$ Constructed as described in Hsu et al (2016). 